\title{
RESIDUALLY SMALL VARIETIES WITH MODULAR CONGRUENCE LATTICES
}

BY

\author{
RALPH FREESE AND RALPH MCKENZIE ${ }^{1}$
}

\begin{abstract}
We focus on varieties $\mathfrak{V}$ of universal algebras whose congruence lattices are all modular. No further conditions are assumed. We prove that if the variety $\mathfrak{V}$ is residually small, then the following law holds identically for congruences over algebras in $\mathcal{V}: \beta \cdot[\delta, \delta]<[\beta, \delta]$. (The symbols in this formula refer to lattice operations and the commutator operation defined over any modular variety, by Hagemann and Herrmann.) We prove that a finitely generated modular variety $\mathfrak{V}$ is residually small if and only if it satisfies this commutator identity, and in that case $\mathfrak{V}$ is actually residually $<\boldsymbol{n}$ for some finite integer $n$. It is further proved that in a modular variety generated by a finite algebra $A$ the chief factors of any finite algebra are bounded in cardinality by the size of $A$, and every simple algebra in the variety has a cardinality at most that of $\boldsymbol{A}$.
\end{abstract}

By a variety we mean a class of similar algebras closed under the formation of subalgebras, homomorphic images and direct products. A variety is locally finite if every finitely generated algebra in it is finite. A variety $V(A)=H S P(A)$ generated by a finite algebra $A$ is locally finite. If $A$ is an infinite locally finite subdirectly irreducible algebra, then $H S(A)$ includes finite subdirectly irreducible algebras with no finite bound on their cardinality. (A theorem of Quackenbush [17].) Thus for a locally finite variety $\mathfrak{V}$ there are exactly two possibilities: either there is an integer $n$ such that every finite subdirectly irreducible algebra in $\mathcal{V}$ has size at most $n$, and then every subdirectly irreducible algebra in $\mathcal{V}$ has size at most $n$, or else $\mathcal{V}$ has finite subdirectly irreducible algebras with no finite bound on their size. In the latter case there are still many possibilities for the spectrum of subdirectly irreducible algebras in $\mathfrak{V}$. There may or may not be an infinite subdirectly irreducible algebra. A variety is called residually $<\kappa$ (where $\kappa$ is a cardinal, possibly finite) if every subdirectly irreducible algebra in it has cardinality $<\kappa$. The only known example of a locally finite, residually $<\omega$ (i.e. residually finite) variety that is not residually $<n$ for any $n<\omega$ is due to Baldwin and Berman [2]-their variety has infinitely many basic operations and is not generated by any one finite algebra.

\footnotetext{
Received by the editors January 10, 1980. Presented to the Society on March 28 and 29, 1980 under the titles Varieties with modular congruences and Narrow varieties, and a characterization of finitely generated, decidable, modular varieties.

1980 Mathematics Subject Classification. Primary 08A15, 06A30; Secondary 20D99.

Key words and phrases. Residually small variety, subdirect product, modular congruence lattice, commutator of congruences, chief factors of universal algebras, groups with abelian Sylow subgroups.

${ }^{1}$ Research of the first author supported by NSF Grant MCS 77-01933 and research of the second author supported by NSF Grant MCS 77-22913.
} 
We shall call $\mathcal{V}$ res. $\ll \omega$ if for some $n<\omega$ it is res. $<n$. Quackenbush [17] asked whether there exists a finite algebra $A$ such that $V(A)$ is res. $<\omega$ and not res. $\ll \omega$. Taylor [22] proved that no such $A$ can be of finite type and generate a variety with regular and permutable congruences. Freese (unpubiished) dropped one of Taylor's assumptions (permutability). A variety with regular congruences also has modular congruence lattices. The main result of this paper is a considerable improvement on Taylor's and Freese's earlier results. We prove (Theorem 8 ) that if $A$ is any finite algebra (not necessarily of finite type) and $V(A)$ is congruence modular, then $V(A)$ is res. $\ll \omega$ if and only if it is residually small (res. $<\kappa$ for some cardinal $\kappa$ ). Actually, our theorem gives an intrinsic characterization of those finite algebras $A$ in any fixed congruence modular variety $\mathcal{V}$ which generate a res. $\ll \omega$ subvariety, and the characterization shows that they form a local subvariety of $\mathcal{V}$ (closed under finite products as well as homomorphic images and subalgebras). A finite group $G$ has this property just in case all the Sylow subgroups of $G$ are abelian. (This was essentially known from the literature-see §5.)

The second author has very recently shown that the algebra in Quackenbush's question cannot be a finite semigroup [13].

This whole paper relies heavily on the rather deep theory of commutators of congruences developed first by J. D. H. Smith [20] for a general variety with permuting congruences, and in the past few years by the Darmstadt school, in particular by Christian Herrmann and Joachim Hagemann, for any variety whose congruence lattices are modular. (See [6]-[10], particularly [9] and [10].) The commutator is a binary operation on congruence lattices which in the case of normal subgroups $B$ and $D$ of a group $G$ specializes to the ordinary commutator group $[B, D]$ generated by the commutator elements $[b, d]$, with $b \in B, d \in D$.

The first section of our paper reviews the theory of commutators, but is not selfcontained. The reader wishing to fully understand our proof will have to read first $\mathrm{HH}[\mathbf{9}]$ and $\mathrm{H} \mathrm{[10].}$

In the second section we present a certain identity for congruences expressed in the join and meet and commutator operations. In the third section we show that this identity is satisfied by all residually small, congruence modular varieties. In the fourth section we show that if $A$ is a finite algebra in a congruence modular variety and if the congruence lattice of each subalgebra of $A$ satisfies the commutator identity then $V(A)$ is residually $\ll \omega$.

The fifth section relates our results to the known facts for groups and modules and expresses some open questions. The final section extends the result of Nation and Taylor bounding chief factors to the congruence modular case. It is also shown that if $A$ is a finite algebra in a congruence modular variety and $B \in V(A)$ is simple then $|B| \leqslant|A|$.

The authors would like to thank Cliff Bergman, Bob Colby, Lee Lady, and Bill Lampe for helpful discussions.

1. Commutator lemmas. Let $\mathfrak{V}$ be a variety with modular congruences. We let 0 and 1 be the least and greatest congruences of any algebra under consideration. For a homomorphism $f: A \rightarrow B$ and congruence $\alpha \in \operatorname{con} A$ and $\beta \in \operatorname{con} B$ we 
write $\hat{f}(\beta)$ for $\{(x, y) \in A \times A:(f(x), f(y)) \in \beta\}$ and $\check{f}(\alpha)$ for $\{(f(x), f(y)):(x, y)$ $\in \alpha\}$. Sometimes we shall write $\hat{\beta}$ and $\check{\alpha}$ for $\hat{f}(\beta)$ and $\check{f}(\alpha)$. Notice that $\hat{f}(\beta)$ is a congruence on $A$, while $\check{f}(\alpha)$ is a congruence on $B$ provided $f$ is onto $B$ and $\alpha \geqslant \operatorname{ker}(f)=\hat{f}\left(0_{B}\right)$. Again let $\alpha \in \operatorname{con} A$ and $\beta \in \operatorname{con} B$. Then $\alpha_{0}$ on $C \subseteq A \times B$ is $\hat{p}_{0}(\alpha)$ and $\beta_{1}=\hat{p}_{1}(\beta)$, where $p_{0}$ and $p_{1}$ are the projection maps into $A$ and $B . \eta_{0}$ and $\eta_{1}$ always denote $\hat{p}_{0}(0)$ and $\hat{p}_{1}(0)$. This is generalized in the obvious way to subalgebras of direct products with more than two components.

Our main tool will be the Herrmann-Hagemann theory of commutators of congruences in a congruence modular variety (see HH [9]). Suppose $A \in \mathcal{V}$ and $\alpha, \beta, \gamma \in \operatorname{con} A$ with $\alpha \leqslant \beta \cdot \gamma$. We say that $\beta$ centralizes $\gamma$ modulo $\alpha$ provided there is a congruence $\kappa$ on $\beta$ (considered as a subalgebra of $A \times A$ ) satisfying

$$
\begin{gathered}
\kappa \cdot \alpha_{0}=\kappa \cdot \alpha_{1}=\alpha_{0} \cdot \alpha_{1}, \\
\kappa+\eta_{i}=\gamma_{i}, \quad i=0,1 .
\end{gathered}
$$

Herrmann and Hagemann show that there is a least $\alpha$ such that $\beta$ centralizes $\gamma$ modulo $\alpha$. This $\alpha$ is denoted [ $\beta, \gamma$ ].

We require the following nontrivial facts from $\mathrm{HH}$ [9].

Proposition 1. For $\alpha, \beta, \gamma, \delta, \gamma_{i} \in \operatorname{con} A, i \in I$ :

(1) $[\beta, \gamma]=[\gamma, \beta] \leqslant \beta \cdot \gamma$.

(2) $\left[\beta, \bigvee_{i \in I} \gamma_{i}\right]=\bigvee_{i \in I}\left[\beta, \gamma_{i}\right]$

(3) For $\alpha \leqslant \beta \cdot \gamma$ the following are equivalent:

(i) $[\beta, \gamma] \leqslant \alpha$.

(ii) $\beta$ centralizes $\gamma$ modulo $\alpha$.

(iii) $[\check{\pi} \beta, \check{\pi} \gamma]=0$ in con $A / \alpha$ where $\pi: A \rightarrow A / \alpha$ is natural.

(iv) There is a homomorphism $\phi: B \rightarrow A$ (onto) for some $B \in \mathcal{V}$ and there are congruences $\sigma, \delta$ on $B$ such that $\sigma \cdot \delta \leqslant \hat{\phi} \alpha$ while $\sigma+\hat{\phi} \alpha \geqslant \hat{\phi} \beta$ and $\delta+\hat{\phi} \alpha \geqslant \hat{\phi} \gamma$.

We call $\beta \in \operatorname{con} A$ abelian if $[\beta, \beta]=0 . A$ is abelian if $1_{A}$ is. Herrmann [10] constructs explicitly from Day's Mal'cev terms for congruence modularity a term with three variables $p(x, y, z)$ satisfying the following.

Proposition 2. $\mathcal{V}$ satisfies $p(x, y, y)=x$ and if $\beta$ is an abelian congruence on $A \in \mathcal{V}$ each $\beta$-block $B=x / \beta$ is the underlying set of an abelian group such that $p(a, b, c)=a-b+c$ for each $a, b, c \in B$. If $f$ is $a k$-ary term function on $A$, and $B_{0}, \ldots, B_{k-1}$ are $\beta$-blocks, then $f\left(B_{0} \times \cdots \times B_{k-1}\right)$ is contained in a single $\beta$-block, say $B_{k}$ (since $\beta$ is a congruence). Moreover, for each $\mathbf{x}, \mathbf{y}, \mathbf{z} \in B_{0}$ $\times \cdots \times B_{k-1}, f(\mathbf{x}-\mathbf{y}+\mathbf{z})=f(\mathbf{x})-f(\mathbf{y})+f(\mathbf{z})$.

This is Lemma 4.2 of [10].

The next proposition shows that commutators behave well with respect to homomorphic images. It may be proved by using Step B of the proof of Theorem 1.4 of $\mathrm{HH}[9]$.

Proposition 3. Suppose that $\phi: A \rightarrow B$ is onto, and $\beta, \gamma \in \operatorname{con} B$. Then $\hat{\phi}([\beta, \gamma])=[\hat{\phi} \beta, \hat{\phi} \gamma]+\operatorname{ker} \phi$. 
If $\beta, \gamma \geqslant \theta$ we let $[\beta, \gamma]_{\theta}$ denote $\hat{\pi}[\check{\pi} \beta, \check{\pi} \gamma]$ where $\pi: A \rightarrow A / \theta$ is the natural map. Thus by Proposition $3,[\beta, \gamma]_{\theta}=[\beta, \gamma]+\theta$.

Proposition 4. An abelian congruence on $A$ permutes with every congruence on $A$.

Proof. Let $[\beta, \beta]=0$ and $\theta$ be arbitrary. By Proposition $2, p(x, y, y)=x$ and $p(x, x, y)=y$ when $x \beta y$. Let $x \beta y \theta z$. Then

$$
x=p(y, y, x) \quad \theta p(z, y, x) \quad \beta \quad p(z, x, x)=z .
$$

It follows that $\beta$ and $\theta$ permute. (For stronger results on permutability see [8].)

Corollary 5. If $\theta \geqslant[\beta, \beta]$ then $\theta$ and $\beta$ permute.

We let $\alpha \prec \beta$ indicate that $\beta$ covers $\alpha . \alpha / \beta \searrow \gamma / \delta$ indicates that $\beta+\gamma=\alpha$ and $\beta \gamma=\delta$. We also write $\gamma / \delta \nearrow \alpha / \beta$ and say that $\alpha / \beta$ transposes down to $\gamma / \delta$.

If $B$ is subdirectly irreducible then the monolith of $B$ is the unique minimal nonzero congruence on $B$. This terminology is borrowed from group theory. The corresponding term in the theory of modules is socle, which more generally is defined to be the join of the atoms of $\operatorname{con} B$.

2. A commutator identity. Let $\mathcal{T}$ be a congruence modular variety of algebras and $A \in \mathcal{V}$. Consider the following identity.

(I) $[\mu, \mu] \cdot \nu \leqslant[\mu, \nu]$, for all $\mu, \nu \in \operatorname{con} A$.

The condition (I) is equivalent to each of the following.

(II) $\nu \leqslant[\mu, \mu]$ implies $[\nu, \mu]=\nu$, for all $\mu, \nu \in \operatorname{con} A$.

(III) If $B$ is any subdirectly irreducible homomorphic image of $A$ with monolith $\beta$, and $\mu \in \operatorname{con} B$ with $[\mu, \beta]=0$, then $[\mu, \mu]=0$.

With the aid of Proposition 3 it is easy to see that (III) can be reformulated in con $A$ as follows: if $\theta$ is completely meet irreducible in $\operatorname{con} A$ with unique cover $\psi$, and $\mu \geqslant \theta$ in con $A$ with $[\mu, \psi] \leqslant \theta$, then $[\mu, \mu] \leqslant \theta$.

The proof of the equivalence of (I), (II), and (III) goes as follows. (II) $\rightarrow$ (I). For arbitrary $\mu, \nu \in \operatorname{con} A,[\mu, \mu] \cdot \nu \leqslant[\mu, \mu]$. Hence by (II) $[\mu, \mu] \cdot \nu=[[\mu, \mu] \cdot \nu, \mu]$ $\leqslant[\nu, \mu]$. (I) $\rightarrow$ (III). Let $\theta$ be completely meet irreducible in $\operatorname{con} A$ with unique cover $\psi$ and suppose $[\mu, \psi] \leqslant \theta$ for some $\mu \geqslant \theta$. (I) yields $[\mu, \mu] \cdot \psi \leqslant[\mu, \psi] \leqslant \theta$. Hence $\theta=\theta+[\mu, \mu] \cdot \psi=\psi(\theta+[\mu, \mu])$. Since $\theta$ is meet irreducible this implies $[\mu, \mu] \leqslant \theta$. (III) $\rightarrow$ (II). Suppose (II) fails. Then there are $\mu, \nu \in \operatorname{con} A$ with $\nu \leqslant$ $[\mu, \mu]$ and $[\mu, \nu]<\nu$. This latter inequality implies that there is a completely meet irreducible $\theta$ with $\theta \geqslant[\mu, \nu]$ but $\theta \neq \nu$. Let $\psi$ be the unique cover of $\theta$. Since $\psi \leqslant \theta+\nu,[\theta+\mu, \psi] \leqslant[\theta+\mu, \theta+\nu] \leqslant[\mu, \nu]+\theta \leqslant \theta$. Hence by (III) $[\theta+\mu, \theta$ $+\mu] \leqslant \theta$. But this implies $\nu \leqslant[\mu, \mu] \leqslant[\theta+\mu, \theta+\mu] \leqslant \theta$, contrary to the choice of $\theta$.

We shall say that $A$ satisfies (II $)_{s}$ if every subalgebra of $A$ satisfies (II).

THEOREM 6. The class of algebras in $\mathfrak{V}$ satisfying $\left(\mathrm{II}_{s}\right)$ is closed under $H, S$, and finite products.

Proof. For $S$ it is clear. For $H$, let $A$ satisfy $\left(\mathrm{II}_{s}\right)$ and $C \subseteq A / \theta$ for some $\theta \in \operatorname{con} A$. Then $C=B / \gamma$ where $B \subseteq A$. If (II) fails for $C$ then there are $\mu, \nu \in \operatorname{con} B, \mu, \nu \geqslant \gamma$, and using Proposition $3, \nu \leqslant[\mu, \mu]_{\gamma}=[\mu, \mu]+\gamma$ while 
$[\nu, \mu]_{\gamma}=[\nu, \mu]+\gamma<\nu$. Then $\nu \leqslant \mu$ and $[\nu, \mu] \ngtr[\mu, \mu] \cdot \nu$, else $[\nu, \mu]+\gamma>[\mu, \mu]$ $\cdot \nu+\gamma \geqslant \nu$. Thus (I) fails in con $B$, a contradiction.

For products, suppose that $C=A \times B$ where $A, B$ satisfy $\left(\mathrm{II}_{s}\right)$, and $D \subseteq C$. We can assume that $A=p_{0} D, B=p_{1} D$. Let $\eta_{0}$ and $\eta_{1}$ be the projection kernels, and suppose that $\nu \leqslant[\mu, \mu], \nu, \mu \in \operatorname{con} D$. Then $\nu+\eta_{0}<\left[\mu+\eta_{0}, \mu+\eta_{0}\right]_{\eta_{0}}$. Since $A$ satisfies (II), then

$$
\nu+\eta_{0}=\left[\nu+\eta_{0}, \mu+\eta_{0}\right]_{\eta_{0}}=[\nu, \mu]+\eta_{0} .
$$

Thus $\nu=\nu \cdot\left([\nu, \mu]+\eta_{0}\right)=[\nu, \mu]+\eta_{0} \cdot \nu$. Likewise $\nu=[\nu, \mu]+\eta_{1} \cdot \nu$.

By the same token,

and

$$
\eta_{1} \cdot \nu+\eta_{0}=\left[\eta_{1} \cdot \nu+\eta_{0}, \mu+\eta_{0}\right]_{\eta_{0}}
$$

$$
\eta_{1} \nu \leqslant\left[\eta_{1} \cdot \nu, \mu\right]+\eta_{0}
$$

Thus $\eta_{1} \cdot \nu=\left[\eta_{1} \cdot \nu, \mu\right]+\eta_{0} \cdot \eta_{1} \cdot \nu=\left[\eta_{1} \cdot \nu, \mu\right]$. Therefore

$$
\nu=[\nu, \mu]+\eta_{1} \cdot \nu=[\nu, \mu]+\left[\eta_{1} \cdot \nu, \mu\right]=[\nu, \mu] .
$$

\section{Necessity.}

THEOREM 7. Let $\mathfrak{V}$ be a congruence modular variety and suppose there is an $A \in \mathcal{T}$ and $\beta, \gamma \in \operatorname{con} A$ with

$$
\beta \leqslant[\gamma, \gamma] \text { and }[\beta, \gamma]<\beta .
$$

Then $\mathfrak{V}$ is not residually small.

Proof. We shall actually show more: $\mathfrak{V}$ contains subdirect irreducibles with arbitrarily large congruence lattices. The hypotheses say that (II) fails for $\boldsymbol{A}$ and hence (III) fails for $A$. Thus we may assume that $A$ is subdirectly irreducible and that $\beta$ is the monolith of $A$.

Since $[\beta, \gamma]=0$, we have by definition that there is a $\kappa \in \operatorname{con} \gamma$ such that $\kappa \cdot \eta_{0}=\kappa \cdot \eta_{1}=\eta_{0} \cdot \eta_{1}=0$ and $\kappa+\eta_{i}=\beta_{i}, i=0,1$. It is easy to check that $\left\{0, \beta_{0} \cdot \eta_{1}, \kappa, \eta_{0} \cdot \beta_{1}, \beta_{0} \cdot \beta_{1}\right\}$ forms a sublattice isomorphic to $M_{3}$; see Figure 1.

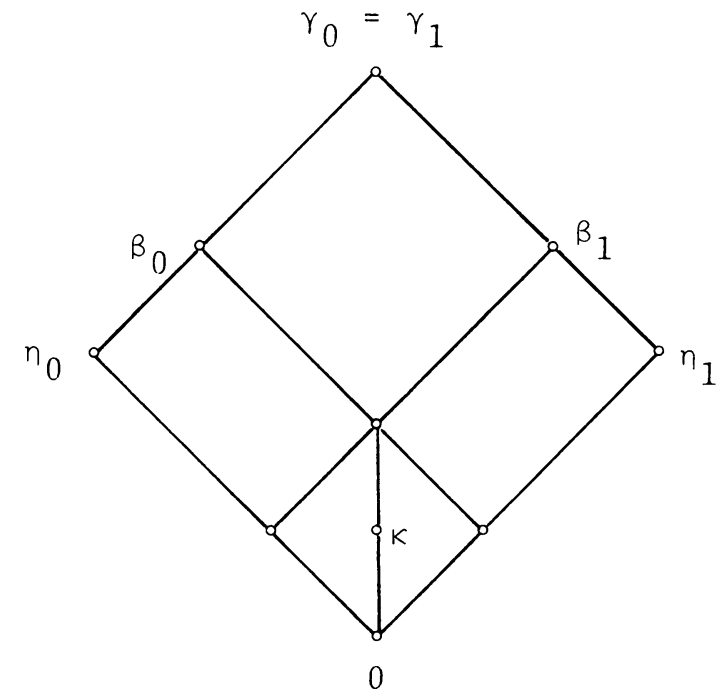

FIGURE 1 
Let $\boldsymbol{\aleph}$ be an arbitrary cardinal and let $B=\left\{\left(a_{\delta}\right) \in{ }^{*} A: a_{\delta} \gamma a_{\varepsilon}\right.$ for all $\left.\delta, \varepsilon<\aleph\right\}$. Recall that if $\theta \in \operatorname{con} A$ then $\theta_{\varepsilon} \in \operatorname{con} B$ is defined by $\left(a_{\delta}\right) \theta_{\varepsilon}\left(b_{\delta}\right)$ if and only if $a_{\varepsilon} \theta b_{\varepsilon}$. Notice that $\gamma_{\delta}=\gamma_{\varepsilon}$ for all $\varepsilon, \delta<\boldsymbol{N}$. We shall denote this congruence again by $\gamma$. Eacy calculations show that $\eta_{\varepsilon}+\eta_{\delta}=\gamma$, for all $\delta \neq \varepsilon$; cf. [5]. Now if $\delta \neq 0$, $B / \eta_{0} \cdot \eta_{\delta}$ is isomorphic to the algebra $\gamma\left(\subseteq^{2} A\right)$ and thus the interval $\gamma / \eta_{0} \cdot \eta_{\delta}$ contains a copy of the lattice of Figure 1. Thus there is a $\kappa_{\delta} \in \operatorname{con} B$ with $\kappa_{\delta} \cdot \eta_{0}=\kappa_{\delta} \cdot \eta_{\delta}=\eta_{0} \cdot \eta_{\delta}$ and $\kappa_{\delta}+\eta_{0}=\beta_{0}, \kappa_{\delta}+\eta_{\delta}=\beta_{\delta}$. Let $\eta_{\delta}^{\prime}=\bigwedge_{e \neq \delta} \eta_{\varepsilon}, \kappa_{\delta}^{\prime}=$ $\kappa_{\delta}\left(\eta_{0}^{\prime}+\eta_{\delta}^{\prime}\right)$, and $\theta_{\delta}=\eta_{\delta}^{\prime} \beta_{\delta}$.

Easy calculations show that $\left\{\eta_{\delta}^{\prime}\right\}$ is an independent set (i.e. $\eta_{\delta}^{\prime}\left(\eta_{\varepsilon_{1}}^{\prime}+\cdots+\eta_{e_{n}}^{\prime}\right)$ $=0$ if $\left.\delta \notin\left\{\varepsilon_{1}, \ldots, \varepsilon_{n}\right\}\right)$ and

$$
\begin{aligned}
\gamma / \eta_{\delta} & \searrow \eta_{\delta}^{\prime} / 0 \quad \text { all } \delta, \\
\gamma / \eta_{0} \cdot \eta_{\delta} & \searrow \eta_{0}^{\prime}+\eta_{\delta}^{\prime} / 0 \quad \text { all } \delta \neq 0 .
\end{aligned}
$$

Since $\theta_{\delta} \leqslant \eta_{\delta}^{\prime},\left\{\theta_{\delta}\right\}$ is an independent set and, since $\beta_{\delta}$ is the unique cover of $\eta_{\delta}$ and $\beta_{\delta} \leqslant \gamma, \theta_{\delta}$ is the unique atom below $\eta_{\delta}^{\prime}$. Also note

$$
\eta_{0} \beta_{\delta}\left(\eta_{0}^{\prime}+\eta_{\delta}^{\prime}\right)=\beta_{\delta}\left(\eta_{0} \eta_{0}^{\prime}+\eta_{\delta}^{\prime}\right)=\beta_{\delta} \eta_{\delta}^{\prime}=\theta_{\delta}
$$

and similarly $\beta_{0} \eta_{\delta}\left(\eta_{0}^{\prime}+\eta_{\delta}^{\prime}\right)=\theta_{0}$, and $\kappa_{\delta}\left(\eta_{0}^{\prime}+\eta_{\delta}^{\prime}\right)=\kappa_{\delta}^{\prime}$. Since (2) gives a lattice isomorphism of the two quotients and $\left\{\eta_{0} \cdot \eta_{\delta}, \eta_{0} \cdot \beta_{\delta}, \kappa_{\delta}, \beta_{0} \eta_{\delta}, \beta_{0} \beta_{\delta}\right\} \cong M_{3}$ we have $\left\{0, \theta_{0}, \kappa_{\delta}^{\prime}, \theta_{\delta}, \theta_{0}+\theta_{\delta}\right\} \cong M_{3}$ for $\delta \neq 0$.

Define $\theta=\bigvee_{\delta} \theta_{\delta}$ and $\kappa=\bigvee_{\delta>0} \kappa_{\delta}^{\prime}$. Then if $\delta \neq 0 \neq \varepsilon$ are arbitrary,

$$
\kappa+\theta_{\delta}=\kappa+\kappa_{\varepsilon}^{\prime}+\kappa_{\delta}^{\prime}+\theta_{\delta}=\kappa+\kappa_{\varepsilon}^{\prime}+\theta_{0}+\theta_{\delta}=\kappa+\theta_{\varepsilon}+\theta_{0}+\theta_{\delta} \geqslant \theta_{\varepsilon}+\theta_{0} \text {. }
$$

Thus $\kappa+\theta_{\delta}=\theta$ and this also holds if $\delta=0$. Moreover $\theta_{\delta} \$ \kappa$; for otherwise, since $\theta_{\delta}$ is an atom and so compact, $\theta_{\delta} \leqslant \kappa_{\varepsilon_{1}}^{\prime}+\cdots+\kappa_{\varepsilon_{n}}^{\prime}$. First consider the case $\delta=0$. We induct on $n . n=1$ is impossible since $\theta_{0} \cdot \kappa_{\varepsilon_{1}}^{\prime}=0$. Now using the induction hypothesis, $\kappa_{\varepsilon_{1}} \leqslant \theta_{0}+\theta_{\varepsilon_{1}}$ and $\kappa_{\varepsilon_{2}}^{\prime}+\cdots+\kappa_{\varepsilon_{n}}^{\prime} \leqslant \theta_{0}+\theta_{\varepsilon_{2}}+\cdots+\theta_{\varepsilon_{n}}$, and the independence of $\left\{\theta_{\delta}\right\}$, we have

$$
\begin{aligned}
\theta_{0} & =\theta_{0}\left(\kappa_{\varepsilon_{1}}^{\prime}+\cdots+\kappa_{\varepsilon_{n}}^{\prime}\right)=\theta_{0}\left(\theta_{0}+\theta_{\varepsilon_{1}}\right)\left(\kappa_{\varepsilon_{1}}^{\prime}+\cdots+\kappa_{\varepsilon_{n}}^{\prime}\right) \\
& =\theta_{0}\left(\kappa_{\varepsilon_{1}}^{\prime}+\left(\theta_{0}+\theta_{\varepsilon_{1}}\right)\left(\theta_{0}+\theta_{\varepsilon_{2}}+\cdots+\theta_{\varepsilon_{n}}\right)\left(\kappa_{\varepsilon_{2}}^{\prime}+\cdots+\kappa_{\varepsilon_{n}}^{\prime}\right)\right) \\
& =\theta_{0}\left(\kappa_{\varepsilon_{1}}^{\prime}+\theta_{0}\left(\kappa_{\varepsilon_{2}}^{\prime}+\cdots+\kappa_{\varepsilon_{n}}^{\prime}\right)\right)=\theta_{0} \kappa_{\varepsilon_{1}}^{\prime}=0 .
\end{aligned}
$$

The case of $\delta \neq 0$ is easier and left to the reader.

It follows that $\kappa<\theta$. Let $\lambda \in \operatorname{con} B$ be a completely meet-irreducible element such that $\lambda \geqslant \kappa$ and $\lambda \neq \theta$ (cf. 6.2 of [3]). Then $\lambda \eta_{\delta}^{\prime}=0$ for otherwise $\lambda \geqslant \theta_{\delta}$ since $\theta_{\delta}$ is the unique atom below $\eta_{\delta}^{\prime}$. But then $\lambda \geqslant \kappa+\theta_{\delta}=\theta$, a contradiction. Thus $\eta_{\delta}+\eta_{\delta}^{\prime}=\gamma$ and $\lambda \eta_{\delta}^{\prime}=0$. If in addition we had $\lambda+\eta_{\delta} \geqslant \gamma$ then Proposition 1(3(iv)) would imply that, in con $A,[\gamma, \gamma]=0$, contradicting $\beta \leqslant[\gamma, \gamma]$ in con $A$. Hence for each $\delta, \lambda+\eta_{\delta} \ngtr \gamma$. Since $\eta_{\delta}+\eta_{\varepsilon}=\gamma$ for $\delta \neq \varepsilon$, the $\lambda+\eta_{\delta}, \delta<\kappa$, must be pairwise distinct. Hence $|\operatorname{con}(B / \lambda)| \geqslant \aleph$, proving the theorem.

\section{Sufficiency.}

THEOREM 8. The following are equivalent for an algebra $A$ in a congruence modular variety with $|A|=m<\omega$.

(1) $V(A)$ is residually small.

(2) $V(A)$ is residually $\leqslant(l+1) ! m$ where $l=m^{m^{m+1}}$.

(3) For any $\mu, \nu \in$ con $C$ where $C \subseteq A, \nu \leqslant[\mu, \mu]$ implies $\nu=[\mu, \nu]$. 
Proof. Theorem 7 gives (1) implies (3), and (2) implies (1) trivially.

For the rest of this section we assume that (3) holds. By Quackenbush's theorem [17] it will suffice to show that every finite subdirect irreducible in $V(A)$ has cardinality bounded as stated in (2). By the results of $\$ 2$ we know that (II) holds in every finite algebra of $V(A)$.

LEMMA 9. Every finite subdirectly irreducible algebra $B$ in $V(A)$ has an abelian congruence $\gamma$ such that $B / \gamma \in H S(A)$.

Proof. If $B \in H S(A)$, take $\gamma=0$. Otherwise write $B \cong B^{\prime}=C / \theta$ where $C \subseteq$ $A_{0} \times A_{1} \times \cdots \times A_{k}, A_{i} \in H S(A)$, and $k$ is minimal. We can assume that $C$ projects onto each $A_{i}$. Let $\eta$ be the kernel of the projection onto $A_{0}$, and $\eta^{\prime}$ the kernel of the projection into $A_{1} \times \cdots \times A_{k}, \eta, \eta^{\prime} \in \operatorname{con} C$. Let $\psi$ be the unique cover of $\theta$ in con $C$.

Now $\eta^{\prime} \$ \theta$, by minimality. Hence

$$
[\psi, \theta+\eta] \leqslant\left[\theta+\eta^{\prime}, \theta+\eta\right] \leqslant \theta+\eta \cdot \eta^{\prime}=\theta .
$$

Let $\beta$ and $\gamma$ be the congruence on $B$ corresponding to $\psi$ and $\theta+\eta$. Thus $\beta$ is the monolith of $B$ and $B / \gamma \in H\left(A_{0}\right) \subseteq H S(A)$. The above inequality implies that $0=[\beta, \gamma]$ (Proposition 3). Hence $[\gamma, \gamma] \neq \beta$, as (II) holds in $B$. Thus $[\gamma, \gamma]=0$.

From here on we assume $B$ is a finite subdirectly irreducible algebra in $V(A)$ but not in $H S(A)$. Let $\gamma$ be the congruence given by Lemma 9. Then $\gamma>0$ and so $\gamma \geqslant \beta$, where $\beta$ is the monolith of $B$. Let $C_{1}, \ldots, C_{n}$ be the blocks of $\gamma$. Thus $n \leqslant|A|$. By Proposition 2 each $C_{i}$ is the underlying set of an abelian group and there is a 3 variable term $p$ (not depending on $i$ ) such that $p(x, y, z)=x-y+z$ if $x, y, z \in C_{i}$. Furthermore if $f$ is a $k$ variable term-function then $f$ restricted to $C_{\sigma(1)} \times \cdots \times C_{\sigma(k)}$ maps $C_{\sigma(1)} \times \cdots \times C_{\sigma(k)}$ into some $\gamma$-block, say $C_{j}$, since $\gamma$ is a congruence. Moreover, $f(\mathbf{x}-\mathbf{y}+\mathbf{z})=f(\mathbf{x})-f(\mathbf{y})+f(\mathbf{z})$ for $\mathbf{x}, \mathbf{y}, \mathbf{z} \in C_{\sigma(1)}$ $\times \cdots \times C_{\sigma(k)}$. Let $0_{i}$ be the null element of $C_{i}$.

Lemma 10. If $g(x)=f\left(x, c_{1}, \ldots, c_{k}\right)$ is a unary algebraic function mapping $C_{i}$ into $C_{j}$, then there is an $n+1$ variable term $t\left(x, x_{1}, \ldots, x_{n}\right)$ and an element $c \in C_{j}$ such that for all $x \in C_{i}$

$$
g(x)=t\left(x, 0_{1}, \ldots, 0_{n}\right)+c .
$$

Proof. Let $c_{r} \in C_{\sigma(r)}, \quad r=1, \ldots, k$, and set $\sigma(0)=i$. Let $\mathbf{0}=$ $\left(0_{\sigma(0)}, 0_{\sigma(1)}, \ldots, 0_{\sigma(k)}\right)$ and let $\mathbf{y}=\left(y_{0}, y_{1}, \ldots, y_{k}\right)$ and $\mathbf{z}=\left(z_{0}, z_{1}, \ldots, z_{k}\right)$ be elements of $\prod_{r=0}^{k} C_{\sigma(r)}$. Then $f(\mathbf{y}+\mathbf{z})=f(\mathbf{y}-\mathbf{0}+\mathbf{z})=f(\mathbf{y})+f(\mathbf{z})-f(\mathbf{0})$. Thus if $\mathbf{x}=\left(x_{0}, x_{1}, \ldots, x_{k}\right) \in \Pi_{r=0}^{k} C_{\sigma(r)}$, then $f(\mathbf{x})=f\left(x_{0}, 0, \ldots, 0\right)+f\left(0, x_{1}, \ldots, x_{k}\right)$ $-f(0)$. Consequently if $x \in C_{i}$ then $g(x)=f\left(x, 0_{\sigma(1)}, \ldots, 0_{\sigma(k)}\right)+c$ where $c=$ $f\left(0_{\sigma(0)}, c_{1}, \ldots, c_{k}\right)-f(0)$. Set $t\left(x_{0}, x_{1}, \ldots, x_{n}\right)=f\left(x_{0}, x_{\sigma(1)}, \ldots, x_{\sigma(k)}\right)$. Then $g(x)$ $=t\left(x, 0_{1}, \ldots, 0_{n}\right)+c$ for all $x \in C_{i}$.

Let $\theta(a, b)=\beta$ be the monolith of $B$. Since $\theta(a, b)<\gamma, a$ and $b$ are in the same block, say $C_{1}$. Now $a-b=p\left(a, b, 0_{1}\right) \theta(a, b) p\left(a, a, 0_{1}\right)=0_{1}$ shows $\theta\left(a-b, 0_{1}\right)$ $\leqslant \theta(a, b)$ and hence equal. Changing notation, we let $\theta\left(a, 0_{1}\right)$ be the minimal congruence on $B$ with $a \in C_{1}$. 
LeMmA 11. If $c \in C_{i}$ and $c \neq 0_{i}$ then there is a unary algebraic function $g$ on $B$ such that $g\left(0_{i}\right)=0_{1}$ and $g(c)=a$.

Proof. Since $\left(a, 0_{1}\right) \in \theta\left(c, 0_{i}\right)$ there is a sequence $a_{0}=a, a_{1}, \ldots, a_{k}=0_{1}$ of elements of $B$ and unary algebraic functions $g_{0}, \ldots, g_{k-1}$ such that $g_{j}$ maps the set $\left\{c, 0_{i}\right\}$ onto the set $\left\{a_{j}, a_{j+1}\right\}$. Since $c \gamma 0_{i}$ all of the $a_{j}$ are in $C_{1}$. Define $\chi$ : $\{0, \ldots, k-1\} \rightarrow\{ \pm 1\}$ by $\chi(j)=1$ if $g_{j}(c)=a_{j}$ and $\chi(j)=-1$ if $g_{j}(c)=a_{j+1}$. Let $-1 \cdot g_{j}(x)=p\left(0_{1}, g_{j}(x), 0_{1}\right)$, which again is a unary algebraic function. Let $h(x)=\chi(0) g_{0}(x)+\cdots+\chi(k-1) g_{k-1}(x)$, where addition is defined inductively by $h(x)=p\left(\chi(0) g_{0}(x), 0_{1}, \chi(1) g_{1}(x)+\cdots+\chi(k-1) g_{k-1}(x)\right)$. Thus $h(x)$ is a unary algebraic function. Finally let $g(x)=h(x)-h\left(0_{i}\right)\left(=p\left(h(x), h\left(0_{i}\right), 0_{1}\right)\right)$. It is easy to see that this $g(x)$ does the job.

In order to prove the theorem we need to show that $\left|C_{i}\right| \leqslant(l+1)$ ! for $i=$ $1, \ldots, n$. If $g(x)$ is as in Lemma 11 then by Lemma 10 there is an $n+1$ variable term $t$ such that $g(x)=t\left(x, 0_{1}, \ldots, 0_{n}\right)-t\left(0_{i}, 0_{1}, \ldots, 0_{n}\right)$. Of course there are at most $m^{m^{n+1}} \leqslant m^{m^{m+1}}$ inequivalent $n+1$ variable terms for $V(A)$. Thus let $g_{1}, \ldots, g_{k}$ be a list of all unary algebraic functions mapping $C_{i}$ into $C_{1}$ with $g_{j}\left(0_{i}\right)=0_{1}$. Then $k \leqslant m^{m^{m+1}}=l$.

Each $g_{j}$ is an affine map from $C_{i}$ to $C_{1}$. However, since $g_{j}\left(0_{i}\right)=0_{1}$, each $g_{j}$ is a group homomorphism. Now we can associate to each nonzero element $c$ of $C_{i}$ one of the $g_{j}$ such that $g_{j}(c)=a$. We shall show by induction on $k$ that if $G$ is any abelian group for which there is a set of $k$ homomorphisms $\left\{g_{1}, \ldots, g_{k}\right\}$ from $G$ to $C_{1}$ such that for each $x \neq 0$ in $G$ one of these homomorphisms sends $x$ to $a$, then $|G| \leqslant(k+1)$ !. The initial case is left to the reader.

Associate with each nonzero $x \in G$ a $g_{j}$ with $g_{j}(x)=a$. By renumbering we may assume that $g_{1}$ has the most elements of $G$ assigned to it. Let $x_{1}, \ldots, x_{r}$ be these elements. Then $|G| \leqslant 1+r k$. Let $G_{1}$ be the kernel of $g_{1}$. Since $x_{j}-x_{1} \in G_{1}$, $j=1, \ldots, r,\left|G_{1}\right| \geqslant r$. Hence $|G| \leqslant 1+k\left|G_{1}\right|$. Now $G_{1}$ satisfies the hypothesis of the induction using only $\left\{g_{2}, \ldots, g_{k}\right\}$ since $g_{1}\left(G_{1}\right)=0_{1}$. Thus $\left|G_{1}\right|<k$ ! and thus $|G| \leqslant 1+k \cdot k ! \leqslant(k+1)$ !, proving the theorem.

We close this section by showing that, for $C$ properly chosen in the proof of Lemma $9, \theta+\sum \eta_{i}$ is an abelian congruence of $C$. Let $V(A)$ be congruence modular with $A$ finite but not necessarily satisfying condition (3) of Theorem 8 . If $B$ is a finite subdirect irreducible in $V(A)$ then $B=C / \theta$ for some $C \subseteq A^{k}$. Let $p_{0}, \ldots, p_{k-1}$ be the projections from $C$ into $A$. For subsets $U, V$ of a lattice let $U \gg V$ mean for each $u \in U$ there is a $v \in V$ with $u \geqslant v$. Since $C$ is finite there are $\eta_{0}, \ldots, \eta_{m-1} \in$ con $C$ satisfying

(1) $\theta \geqslant \wedge \eta_{i}$

(2) $\left\{\eta_{0}, \ldots, \eta_{m-1}\right\} \gg\left\{\operatorname{ker} p_{0}, \ldots, \operatorname{ker} p_{k-1}\right\}$,

(3) if $T \subseteq$ con $C$ with $T \gg\left\{\eta_{0}, \ldots, \eta_{m-1}\right\}$ and $T$ satisfies (1) then $T \supseteq$ $\left\{\eta_{0}, \ldots, \eta_{m-1}\right\}$.

By replacing $C$ by $C / \wedge \eta_{i}$ we may assume $\wedge \eta_{i}=0$. Let $\eta_{i}^{\prime}=\wedge_{j \neq i} \eta_{j}$. If $\theta \cdot \eta_{i}^{\prime}>0$ then we could replace $\eta_{i}$ by $\eta_{i}+\theta \eta_{i}^{\prime}$, violating (1)-(3). Let $\psi$ be the unique cover of $\theta$ and $\bar{\eta}_{i}$ the unique cover of $\eta_{i}$. If we assume that $B \notin H S(A)$ then it follows that $\psi \leqslant\left(\theta+\eta_{i}\right)\left(\theta+\eta_{i}^{\prime}\right)$ for each $i$. An easy application of Proposition $1(3($ iv $))$ yields that $\left[\eta_{i}, \psi\right] \leqslant \theta$. 
Suppose for some $i$, say $i=0$, that $\left[\theta+\sum \eta_{i}, \overline{\eta_{0}}\right]_{\eta_{0}}=\overline{\eta_{0}}$. By modularity $\overline{\eta_{0}}=\eta_{0}$ $+\eta_{0}^{\prime} \psi$. This implies $\left[\psi \cdot \eta_{0}^{\prime}, \theta+\Sigma \eta_{i}\right]=\psi \cdot \eta_{0}^{\prime}$ for if $\left[\psi \cdot \eta_{0}^{\prime}, \theta+\sum \eta_{i}\right]=0$ (which is the only other possibility since $\psi \cdot \eta_{0}^{\prime}$ is an atom) then $\left[\theta+\sum \eta_{i}, \overline{\eta_{0}}\right]=\left[\theta+\sum \eta_{i}, \eta_{0}\right.$ $\left.+\psi \cdot \eta_{0}^{\prime}\right] \leqslant \eta_{0}+0=\eta_{0}$. Since $\left[\psi \cdot \eta_{0}^{\prime}, \theta\right] \leqslant \theta \cdot \eta_{0}^{\prime}=0, \psi \cdot \eta_{0}^{\prime}=\sum_{i=0}^{m-1}\left[\psi \cdot \eta_{0}^{\prime}, \eta_{i}\right]$. Since $\psi \cdot \eta_{0}^{\prime}$ is an atom, there is an $i$, say $i=1$, with $\psi \cdot \eta_{0}^{\prime}=\left[\psi \cdot \eta_{0}^{\prime}, \eta_{1}\right]$. This together with $\left[\psi, \eta_{1}\right] \leqslant \theta$ gives $\psi \cdot \eta_{0}^{\prime}=0$, a contradiction. Thus we have proved the following.

THEOREM 12. Let $A, B, C, \theta$, and $\eta_{i}$ be as above. Then $\left[\theta+\sum \eta_{i}, \bar{\eta}_{i}\right] \leqslant \eta_{i}$.

COROLlary 13. With notation as above, if A satisfies condition (3) of Theorem 8 then $\theta+\sum \eta_{i}$ is an abelian congruence of $C$.

Proof. By the above theorem and condition (3) of Theorem $8\left[\theta+\Sigma \eta_{i}, \theta+\right.$ $\left.\sum \eta_{i}\right] \leqslant \eta_{j}$ for each $j$. Thus $\left[\theta+\Sigma \eta_{i}, \theta+\sum \eta_{i}\right]=0$.

5. Finite groups and modules. The finite groups whose generated variety is residually small, or residually $\ll \omega$, can be characterized exactly as those which do not possess any nonabelian Sylow subgroup. We shall derive this fact as a corollary of Theorem 8 in this section. This characterization has been known for many years to various people, but apparently was never explicitly stated in the literature. Part of it is in Kovács-Newman [11] and Oates-Powell [15].

Let $\left(\mathrm{I}^{\prime}\right)$ denote the property of having no nonabelian Sylow subgroups. Call a finite algebra $A$ critical if $A \notin H S P\left((H S)^{*}(A)\right)$ where $(H S)^{*}(A)$ is $\{B \in H S(A)$ : $|B|<|A|\}$. Oates-Powell proved that $V(G)$ has only finitely many critical algebras, if $G$ is a finite group. Kovács-Newman proved (among other things) that any finite subdirectly irreducible group satisfying $\left(\mathrm{I}^{\prime}\right)$ is critical. Finally, it is easy to show that if $G$ satisfies $\left(\mathrm{I}^{\prime}\right)$ then so does any finite group in $V(G)$. An integration of these three results gives the earlier known argument that $V(G)$ residually $\ll \omega$ when $G$ is a finite group satisfying $\left(\mathrm{I}^{\prime}\right)$. For the converse, a very special case of the construction we used in Theorem 7 was known, which produced very large subdirectly irreducible groups starting with a given nonabelian, nilpotent (Sylow) group.

We know of no very easy proof for the fact that a finite group satisfies $\left(I^{\prime}\right)$ if and only if each of its subgroups satisfies (I). There is a direct argument however, and here it is.

First, if $P$ is any Sylow subgroup of $G$ and $P$ satisfies (I), then $[P, P]=[P, P] \cdot$ $[P, P] \leqslant[[P, P], P]$. Since $P$ is nilpotent, it follows that $[P, P]=0$, i.e. $P$ is abelian.

Second, suppose that $G$ satisfies ( $\left.\mathrm{I}^{\prime}\right)$, while (I) fails to hold in a subgroup $S$ of $G$. Then $S$ has a subdirectly irreducible homomorphic image $H$ with monolith $M$ and, where $\Gamma$ is the centralizer of $M$ in $H, M \leqslant \Gamma$ and $\Gamma$ is nonabelian. (This is another way of stating the failure of (III) for $S$.) Now the monolith $M$ is abelian $(M \subseteq \Gamma)$, thus it is easily seen to be an elementary abelian $p$-group for some prime number $p$. Since $H$ also satisfies $\left(\mathrm{I}^{\prime}\right), \Gamma$ is not a $p$-group. Let $F$ be minimal among those normal subgroups of $H$ contained in $\Gamma$ which are not $p$-groups. Then $M \subseteq F$, and $M \subseteq P \subseteq F$ for a certain Sylow $p$-group $P$ of $F$. Consider the transfer $t$ of $F$ into 
$P$. This is a homomorphism defined as follows: We choose representatives $x_{0}=$ $1, \ldots, x_{k-1}$ for the left cosets $x \cdot P$ of $P$ in $F$, and we put $t(y)=\Pi_{0}^{k-1} b_{i}$ where $y \cdot x_{i}=x_{j_{i}} \cdot b_{i}\left(b_{i} \in P\right)$ for $i<k$. As $P$ is abelian, $t$ is a homomorphism. For any $g \neq 1, g \in M$, we have $g \cdot x_{i}=x_{i} \cdot g(i<k)$ so $t(g)=g^{k} \neq 1$ as $k=[F: P]$ is relatively prime to $p$. Thus $K=\operatorname{ker} t<F$. Notice that $F / K$ is a $p$-group. Take $F^{\prime}=\bigcap_{h \in H} h \cdot K \cdot h^{-1}$. Then $F^{\prime}$ is a normal subgroup of $H, F^{\prime}<F$, and $F / F^{\prime}$ is a $p$-group. $\left(F / h \cdot K \cdot h^{-1} \cong F / K\right.$ a $p$-group for each $h$.) But then $F^{\prime}$ cannot be a $p$-group, and we have contradicted the minimality of $F$.

The above argument has been adapted from Kovács-Newman [11, p. 249].

It is known (Ol'shanskii [16]) that a finite group $G$ satisfies $\left(\mathrm{I}^{\prime}\right)$ if and only if the quasi-variety generated by $G, S P(G)$, is finitely axiomatizable. That $H S P(G)$ has only finitely many s.i. members (all finite) is equivalent to: $\operatorname{HSP}(G)=S P\left(G^{\prime}\right)$ for some finite $G^{\prime}$. Thus we have a curious property of finite groups: $\operatorname{SP}(G)$ is finitely axiomatizeable if and only if $H S P(G)=S P\left(G^{\prime}\right)$ for a finite $G^{\prime}$.

Question. Do these results generalize in any form to arbitrary finite algebras $A$ with $V(A)$ congruence modular?

Question. Does there exist any locally finite congruence modular variety which is residually finite yet has finite subdirectly irreducible members of unbounded size?

Of course Theorem 8 implies that if $A$ is a finite module then $V(A)$ is residually $\ll \omega$. Moreover if $R$ is a finite ring with unit then the variety of all left $R$-modules is generated by $R$ (as a left $R$-module) and hence is residually $\ll \omega$. These results appear in [18].

We present an example of a finite module $A$ such that $V(A)$ is not residually $\leqslant|A|$ (but of course is residually $\ll \omega$ ). Let $K \subseteq F,[F: K]=2$ be finite fields. Let

$$
R=\left\{\left(\begin{array}{ll}
a & b \\
0 & c
\end{array}\right): a, b \in F, c \in K\right\}
$$

and let $A=\left\{\left(\begin{array}{c}a \\ b\end{array}\right): a \in F, b \in K\right\}, B=\left\{\left(\begin{array}{c}a \\ b\end{array}\right): a, b \in F\right\}$, and $C=\left\{\left(\begin{array}{c}a \\ 0\end{array}\right): a \in F\right\}$ be $R$-modules. $A, B$, and $C$ are subdirectly irreducible modules and each has $C$ as its monolith (i.e. socle). It is easy to see that $B$ is a homomorphic image of $A \times A$. (This example is adapted from [4].)

6. Simple algebras and chief factors. In this section we show that if $A$ is a finite algebra in a congruence modular variety and $B$ is a simple algebra in $V(A)$ then $|B| \leqslant|A|$. More generally, if the length of $\operatorname{con} B$ is $n$ then $|B| \leqslant|A|^{n}$. We also show that if $B$ is finite then the chief factors (defined below) of $B$ are no larger than $|A|$. J. B. Nation and Walter Taylor proved these results assuming permutability [22].

For a class of algebras $\mathscr{K}$ let $P_{u}(\mathscr{K})$ denote the class of ultraproducts of members of $\mathcal{K}$. We begin with the following generalization of Jónsson's Theorem, due to Hagemann and Herrmann.

THEOREM 14. Let $\mathcal{K}$ be a class of algebras lying in a congruence modular variety and let $B$ be a subdirectly irreducible algebra in $V(\mathscr{K})$ with monolith $\mu$. Then $B \in H S P_{u}(\mathscr{K})$ or $[\mu, \mu]=0$ in $\operatorname{con} B$. 
Proof. This is Corollary 3.3 of [9].

If $\alpha \geqslant \beta$ are equivalence relations on a finite set $S$ let $\# \alpha / \beta$ be the maximum number of $\beta$-blocks in a single $\alpha$-block. Notice that (1) if $\gamma \leqslant \beta \leqslant \alpha$ and $\check{\alpha}, \check{\beta}$ are the induced equivalence relations on $S / \gamma$ then $\sharp \check{\alpha} / \check{\beta}=\# \alpha / \beta$; (2) if $\alpha / \beta \searrow \gamma / \delta$ then $\sharp \alpha / \beta \geqslant \# \gamma / \delta$; and (3) if $\alpha / \beta \searrow \gamma / \delta$ and in addition $\beta$ and $\gamma$ permute then $\sharp \alpha / \beta=\# \gamma / \delta$. The first statement is clear. To see (2) note by (1) we may assume $\delta=0$. Let $T$ be an $\alpha$-block and $\alpha_{T}(=1), \beta_{T}, \gamma_{T}$ be the restrictions to $T$. Then $T \subseteq B \times C$ where $B=T / \beta_{T}$ and $C=T / \gamma_{T}$; and $T=B \times C$ if $\beta_{T}$ and $\gamma_{T}$ permute. Thus $\gamma_{T}$ corresponds to the kernel of the second projection and so $\sharp \gamma_{T} / 0 \leqslant|B|$. However $\sharp \alpha_{T} / \beta_{T}=\# 1 / \beta_{T}=|B|$. Of course $\# \gamma / 0$ is the maximum of the $\sharp \gamma_{T} / 0$ for $T$ an $\alpha$-block and similarly for $\sharp \alpha / \beta$. Hence (2) holds. If $\beta$ and $\gamma$ permute then so do $\beta_{T}$ and $\gamma_{T}$. Thus $\sharp \gamma_{T} / 0=\# \alpha_{T} / \beta_{T}$ for each $T$ and (3) follows.

THEOREM 15. Let $A$ be a finite algebra in a congruence modular variety and let $B$ be a finite algebra in $V(A)$. If $\alpha>\beta$ in con $B$ then $\# \alpha / \beta \leqslant|A|$.

Proof. We prove this result by induction on $|B|$. Let $\alpha>\beta$ in con $B$. If $\beta>0$ then with the aid of (1) and induction we have $\sharp \alpha / \beta \leqslant|A|$. Thus assume $\alpha>0$. Choose a meet irreducible $\lambda \in \operatorname{con} B$ with $\lambda \ngtr \alpha$. Then $\lambda+\alpha \succ \lambda$ and $\lambda+\alpha / \lambda\rangle$ $\alpha / 0$. Now (2) and the induction hypothesis yield the conclusion unless $\lambda=0$. Hence we may assume $B$ is subdirectly irreducible with monolith $\alpha$.

Now $B=C / \theta$ where $C \subseteq A^{m}$. Let $\eta$ be the kernel of the projection to the first coordinate, $\eta^{\prime}$ the kernel of the map $C \rightarrow A^{m-1}$ projecting to the other coordinates. By choosing $m$ minimal we may assume $\eta^{\prime} \nless \theta$. Let $\psi$ be the unique cover of $\theta$. By (1) it suffices to show $\# \psi / \theta \leqslant|A|$. Since $\eta^{\prime} \leqslant \theta, \psi \leqslant \eta^{\prime}+\theta$. Thus by modularity $\psi \cdot \eta^{\prime} \$ \theta$. Using this and modularity we have $\psi / \theta \searrow \psi \eta^{\prime} / \theta \eta^{\prime} \nearrow \eta+\psi \eta^{\prime} / \eta+\theta \eta^{\prime}$ $\subseteq 1 / \eta$. Thus if we show that $\theta$ and $\psi \eta^{\prime}$ permute then $\# \psi / \theta \leqslant \# 1 / \eta \leqslant|A|$ by (2) and (3). An easy application of Proposition 1(3(iv)) shows $[\psi, \psi] \leqslant \theta$. Thus $\left[\psi \eta^{\prime}, \psi \eta^{\prime}\right] \leqslant \theta \eta^{\prime}$ and applying Corollary 5 we have that $\psi \eta^{\prime}$ permutes with every congruence which contains $\theta \eta^{\prime}$, in particular, $\theta$.

THEOREM 16. Let $A$ be a finite algebra in a congruence modular variety and let $B \in V(A)$. If the length of $\operatorname{con} B$ is $n$ then $|B| \leqslant|A|^{n}$.

Proof. First we shall show that $B$ must be finite. Induct on $n$. If $B$ is not subdirectly irreducible then it is a subdirect product of two algebras with congruence lattices of smaller length. These algebras would then be finite implying that $B$ is finite. Hence we assume $B$ is subdirectly irreducible with monolith $\mu$. If $B \in H S P_{u}(A)=H S(A), B$ is finite. Hence, by Theorem $14, \mu$ is abelian. By induction $B / \mu$ is finite, i.e., $\mu$ has only finitely many blocks. That $B$ is finite follows just as in the proof of Theorem 8 .

Let $\alpha_{0}=0 \prec \alpha_{1} \prec \cdots \prec \alpha_{n}=1$ in con $B$. Since $B$ is finite, Theorem 15 gives $|B| \leqslant \prod_{i=1}^{n} \sharp \alpha_{i} / \alpha_{i-1} \leqslant|A|^{n}$.

Corollary 17. Let $A$ be as above. If $B$ is simple in $V(A)$, then $|B| \leqslant|A|$.

C. Shallon has given a finite algebra $A$ such that $V(A)$ has arbitrarily large simple algebras [19]. 


\section{REFERENCES}

1. J. T. Baldwin, The number of subdirectly irreducible algebras in a variety. II, Algebra Universalis (to appear).

2. J. T. Baldwin and J. Berman, The number of subdirectly irreducible algebras in a variety, Algebra Universalis 5 (1975), 379-389.

3. P. Crawley and R. Dilworth, Algebraic theory of lattices, Prentice-Hall, Englewood Cliffs, N. J., 1973.

4. V. Dlab and C. M. Ringel, On algebras of finite representation type, J. Algebra 33 (1975), 306-394.

5. R. Freese and B. Jónsson, Congruence modularity implies the Arguesian identity, Algebra Universalis 6 (1976), 225-228.

6. H. P. Gumm, Über die Lösungsmengen von Gleichungssystemen über allgemeinen Algebren, Math. Z. 162 (1978), 51-62.

7. Algebras in permutable varieties: geometrical properties of affine algebras, Algebra Universalis 9 (1979), 8-34.

8. H. P. Gumm and C. Herrmann, Algebras in modular varieties: Baer refinements, cancellation and isotopy, Houston J. Math. 5 (1979), 503-523.

9. J. Hagemann and C. Herrmann, A concrete ideal multiplication for algebraic systems and its relation to congruence distributivity, Arch. Math. 32 (1979), 234-245.

10. C. Herrmann, Affine algebras in congruence modular varieties, Acta Sci. Math. 41 (1979), 119-125.

11. L. G. Kovács and M. F. Newman, On critical groups, J. Austral. Math. Soc. 6 (1966), 237-250.

12. W. A. Lampe and W. Taylor, Simple algebras in varieties (preprint).

13. R. McKenzie, Residually small varieties of semigroups, Algebra Universalis (to appear).

14. R. McKenzie and S. Shelah, The cardinals of simple models for universal theories, Proc. Sympos. Pure Math., vol. 25, Amer. Math. Soc., Providence, R. I., 1974, pp. 53-74.

15. S. Oates and M. B. Powell, Identical relations in finite groups, J. Algebra 1 (1964), 11-39.

16. A. Yu. Ol'shanskii, Conditional identities in finite groups, Sibirsk. Mat. Z. 15 (1974), 1409-1413. (English translation)

17. R. W. Quachenbush, Equational classes generated by finite algebras, Algebra Universalis 1 (1971), 265-266.

18. A. Rosenberg and D. Zelinsky, Finiteness of the injective hull, Math. Z. 70 (1959), 372-380.

19. C. Shallon, Non-finitely based algebras derived from lattices, $\mathrm{Ph}$. D. Thesis, UCLA, 1978.

20. J. D. H. Smith, Mal'cev varieties, Lecture Notes in Math., vol. 554, Springer-Verlag, Berlin, 1976.

21. W. Taylor, Residually small varieties, Algebra Universalis 2 (1972), 33-53.

22. __ Subdirectly irreducible algebras in regular, permutable varieties, Proc. Amer. Math. Soc. 75 (1979), 196-200.

Department of Mathematics, University of Hawail at Manoa, Honolulu, Hawail 96822

Department of Mathematics, Universtty of California, Berkeley, California 94720 